\title{
Mixed Phenotype Acute Leukemia, Not Otherwise Specified, Rare Subtypes
}

National Cancer Institute

\section{Source}

National Cancer Institute. Mixed Phenotype Acute Leukemia, Not Otherwise Specified,

Rare Subtypes. NCI Thesaurus. Code C151990.

A very rare group of mixed phenotype acute leukemias, not otherwise specified in which

the blasts show clear-cut evidence of both B-cell and T-cell lineage or evidence of

trilineage (B-cell, T-cell, and myeloid lineage) assignment. (WHO 2017) 